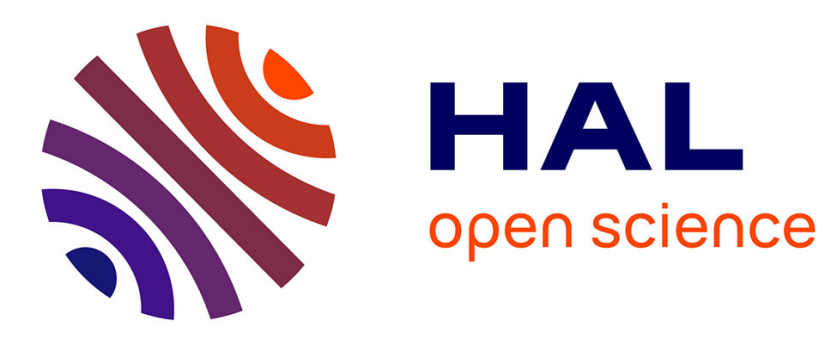

\title{
Wavelet based deconvolution method in ultrasonic tomography
}

Philippe Lasaygues

\section{To cite this version:}

Philippe Lasaygues. Wavelet based deconvolution method in ultrasonic tomography. International congress on ultrasonics, 2007, Vienne, Austria. CD-ROM(4 p.). hal-00440745

\section{HAL Id: hal-00440745 \\ https://hal.science/hal-00440745}

Submitted on 11 Dec 2009

HAL is a multi-disciplinary open access archive for the deposit and dissemination of scientific research documents, whether they are published or not. The documents may come from teaching and research institutions in France or abroad, or from public or private research centers.
L'archive ouverte pluridisciplinaire HAL, est destinée au dépôt et à la diffusion de documents scientifiques de niveau recherche, publiés ou non, émanant des établissements d'enseignement et de recherche français ou étrangers, des laboratoires publics ou privés. 


\title{
Wavelet based deconvolution method in ultrasonic tomography
}

\author{
Philippe Lasaygues
}

Laboratory of Mechanics and Acoustics, UPR CNRS 7051, 13402 Marseille cedex 20, France, lasaygues@1ma.cnrs-mrs.fr

\begin{abstract}
This paper deals with the quantitative and qualitative ultrasonic imaging of media contrasting strongly with the background (bones, for example). The wave propagation in these structures, which involves on several phenomena, generates ultrasound signals forming packages with different time and frequency signatures. To correlate the complex physical processes involved with the various packets forming the signals, it is advisable to separate the useful information (about the various packages, for example) from the parasite information (electronic noise). After testing several possible methods (filtering, deconvolution) with variable levels of success, it was proposed here to increase the resolution of the ultrasound signals, using a method of processing which was optimized by simultaneously analyzing the time and frequency parameters. For this purpose, algorithms based on the wavelet decomposition of the signals were used and suitable transmitted signals correlated with the parameters of the experimental device and the incident wave, were selected. The mathematical properties of the transmitted signal could be adapted themselves well to this time and frequency based approach. This method, which we have called the "wavelet based deconvolution method", makes it possible to determine the transfer function of the scatterer, which we attempted to reconstruct.
\end{abstract}

Key words: Ultrasonic tomography, hard tissues, deconvolution, wavelet transform.

\section{A. Introduction}

Medical imaging is the main field in which ultrasonic computed tomography (UCT) is applied. UCT, which involves the linearisation of the inverse problem of acoustic wave propagation, can be used to detect small perturbations occurring against a reference background. In the case of soft tissues, the mean medium (the background) serves as a homogeneous reference medium, and the waves cross an almost homogeneous and isotropic medium with similar acoustical properties to that of water. When the contrasts are too strong (in the case of bone structures for example), linear theories no longer reasonably apply, and the presence of some physical phenomena associated with the wave propagation makes it necessary to change the methods used for the acquisition of the ultrasonic signals. The more the frequency of the wave increases, the more its propagation will obviously be perturbed. To overcome these problems, the use of low ultrasound frequencies $(<3$ $\mathrm{MHz}$ ) provides an effective possible alternative [1]: when the frequency of the transmitted wave decreases, certain zones turn out to be more homogeneous and the attenuation is also reduced. However, if the depth of the field increases, the resolution of the signals and hence that of the reconstructed images is bound to decrease. Even with low frequencies, the complexity of the wave propagation process generates extremely complex acoustic signals consisting of several packets with different signatures, which it is often difficult to analyze (in terms of the wavepaths, volume, guided or surface waves, and attenuation) and to interpret.

To improve the quality of the signals used either to reconstruct a tomographic image or to characterize the physical processes involved, or both, we focused on the signal processing. In the particular case where ultrasonic imaging is applied to structures, which contrast strongly with the background, optimizing the signal processing used seemed to be a useful approach because tissue characterization requires quantified acoustical information extracted from signals. Several approaches to this problem have been used so far, such as filtering, spectrum analysis and a method involving the deconvolution of the signals using a characteristic transfer function of the experimental device [2]. The latter operation improves the information in the high and low frequency ranges. The alternative method presented in the present study, which is based on a multi-scale decomposition procedure of the signals, makes it possible to process all the information available in terms of frequency and time. But this analysis alone does not suffice to optimize the signal processing because the acoustic signature of the transmitted wave, i.e. the incident wave reaching the scatterer, also has to match some or all of the wavelets' mathematical properties. The main aim of this study is to show the usefulness of this method based on the optimized shaping of the electronically transmitted signal associated with the incident wave propagating through the media, in comparison with the conventional signal processing approach.

\section{B. Acoustical statements}

The frequencies used here $(1 \mathrm{MHz})$ where performing UCT on strongly contrasted materials are therefore lower than those formerly used in practice (for clinical and other purposes, > $3 \mathrm{MHz}$ ). This increases the wavelength of the transmitted wave $(1.5 \mathrm{~mm}$ in water) and decreases the quantity of energy lost during the propagation of the wave. It also increases the depth of penetration of the wave into the medium. The scatterer analyzed will be then taken to consist of a quasi-homogeneous medium. But this reduces the accuracy of the separating power and finally 
the resolution decreases. It is then more difficult on the tomogram to identify details such as boundaries (see Fig.1).
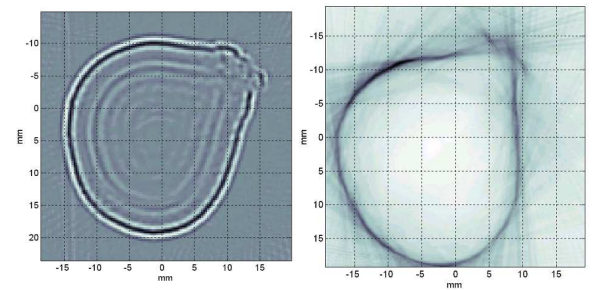

Fig.1 Effects of the frequency on the resolution of ultrasonic computed tomography performed on adult human thighbone (left) $500 \mathrm{kHz}$, (right) $2.25 \mathrm{MHz}$.

The propagation of the waves will result in signals with specific characteristics, which will depend on the pathways taken and on the mode of data acquisition (reflection, transmission or diffraction) used. The following examples (see Fig.2) show how difficult it is to analyse the acoustical signatures. In this example, working in the transmission mode (but similar complexity occurs in the reflection and diffraction modes), a different signature and different packets can be detected, depending on whether the wave is propagating through a solid region (2), a medium solid region (1) comparable to a multi-layer (solid/fluid/solid) material, or the region near the border contrasting with the background (3).
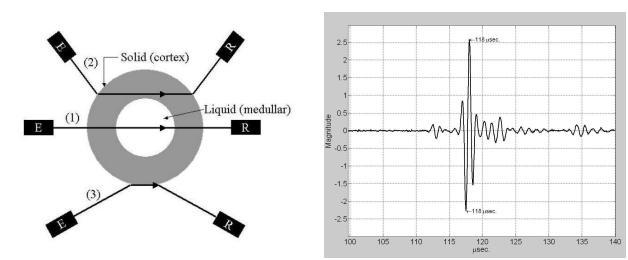

(zone 1)

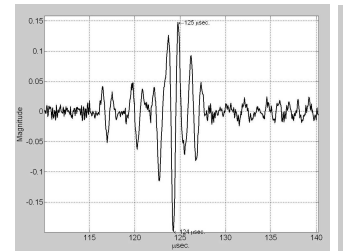

(zone 2)

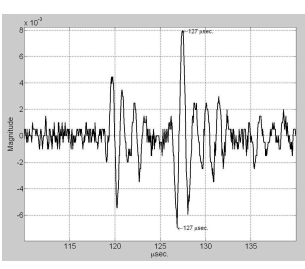

(zone 3)
Fig.2. Ultrasonic signals depending on the bone region involved: (1) multi-layer solid/fluid/solid (2) solid (3) limit contrasting with the background

In the case of objects showing a strong contrast with the background, a large proportion of the incident energy is scattered (or simply reflected) and the transmitted proportion is refracted (deviated) after modal conversion at the interface between the water/scatterer/central cavity, at various angles. The diffraction will not be isotropic. The various wave packets constituting the signals depend decisively on the pathway taken and on the nature of the waves (compressional or shear waves, surface or guided waves). The idea of using a specific signal processing method is to extract as much information as possible about these various packets by analyzing the time and frequency signatures of the signals. It is therefore necessary to decompose these signals so as to identify their constituents.

Wavelet transformation makes it possible to process a signal and to analyze the very local, and much more global, frequency and time parameters. Based on this transformation, a time versus scale diagram can be obtained, giving the evolution of the frequencies with time. Like time-scale processing, wavelet decomposition lends itself very well to detecting and discriminating between signals during the data pre-processing phase as well as the filtering step carried out during the image reconstruction phase.

\section{Material and methods}

\section{The test bench}

The test bench we have developed consists in a main symmetrical arm carrying two secondary transversal arms. Each arm supports an aligned transducer, which can be moved linearly or turned.

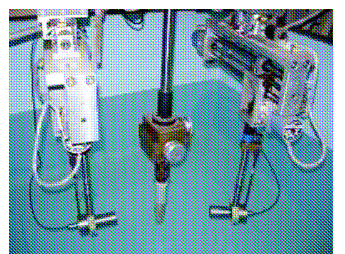

Fig.3. Ultrasonic control of a high-contrast scatterer

The electro-acoustic device and the transducers therefore serve as a continuous, linear stationary causal filter. The input and output signals are connected by convolution:

$$
s(t)=\left(x \otimes h_{M}\right)(t)=\left(h_{T} \otimes e \otimes h_{M}\right)(t),
$$

where $e(t)$ is the electric signal conveyed to the transmitter, $h_{T}(t)$ is the pulse response of the two transducers (which is assumed to be known and to be identical), and $h_{M}$ is the pulse response of the scatterer.

When the input $x(t)$ cannot be reasonably approximated by a Dirac function, the function $h_{M}(t)$ has to be deduced from the output measurement $\mathrm{s}(t)$ and then we must deal with deconvolution.

\section{C.1. Wavelet based deconvolution}

Since $e(t)$ is generally comparable to a unit impulse function (i.e. a Dirac delta function), the input will be equal to the transducer's response $x(t) \approx h_{T}(t)$. Let us now generate a wave corresponding to a transmitted signal $x(t)$ having suitable properties for specific processing method, such as wavelet analysis.

If $x(t)$ is a wavelet denoted $\varphi_{J}(t)$ which is centered on the (dilation) scale denoted $\mathrm{J}(\mathrm{J} \in \mathrm{Z})$, we have:

$$
s(t)=\varphi_{J}(t) \otimes h_{M}(t),
$$

Time-scale decomposition of the signal $s(t)$ on a dyadic grid [4-5] makes it possible to calculate the coefficients $X_{j}$,

$$
X_{j}(t)=s(t) \otimes \phi_{j, 0}(t)=h_{M} \otimes\left(\varphi_{J} \otimes \phi_{j, 0}\right)(t)
$$

where $\phi_{j, 0}(t)$ is a wavelet centered on the scale $\mathrm{j}$ 
( $j \in Z$ ). The properties of the wavelet decomposition (an orthogonal decomposition in this case) are such that the coefficients, $X j$ nullify everywhere except for $\mathrm{j}=\mathrm{J}$, which makes it possible to directly determine the response of the scatterer without encountering any further filtering effects.

$$
X_{j}(t)=\left\{\begin{array}{l}
\left(h_{M} \otimes \delta\right)(t)=h_{M}(t) \text { if } j=J \\
0 \quad \text { if } j \neq J
\end{array},\right.
$$

If it is possible to process the transfer function of the experimental device in such a way that it is identical to a wavelet function, this algorithm directly yields the response of the scatterer.
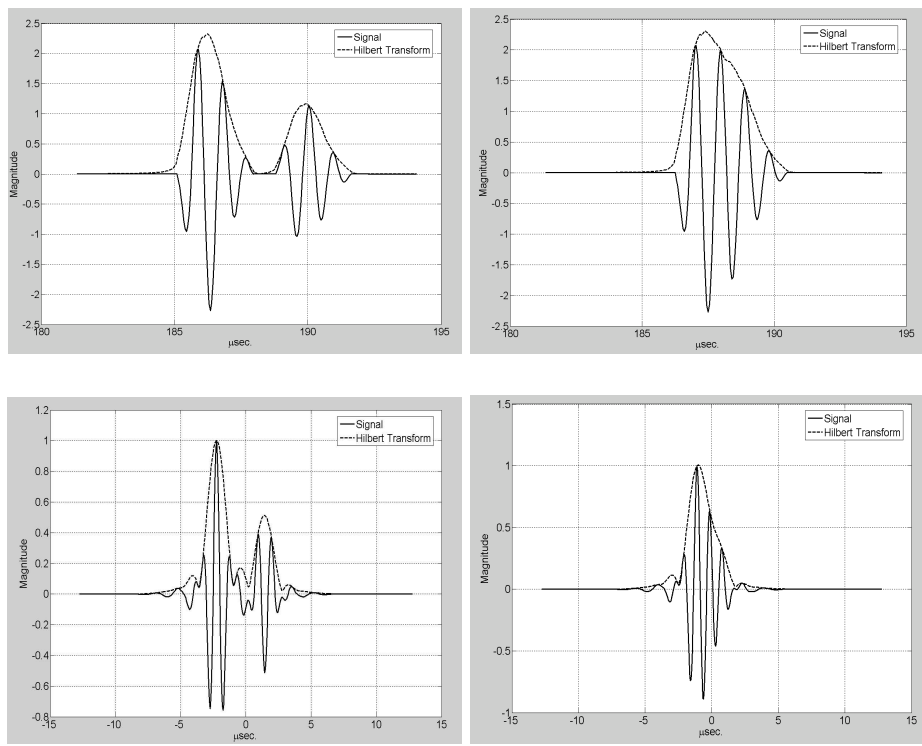

Fig.4. Simulated ultrasonic signals transmitted in plane interfaces at various depths D (left) $5 \mathrm{~mm}$, (right) $2 \mathrm{~mm}$; (height), pulse echo (bottom), wavelet at $\mathrm{J}=-4,512$ samples, frequency rate $20 \mathrm{MHz}$, velocity of compression waves $=2700 \mathrm{~m} / \mathrm{s}$

These signals were then decomposed into wavelet functions (several scales $\mathrm{j}$ ). The maximum coefficients of

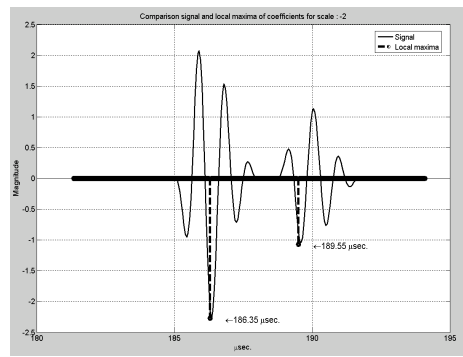

the dyadic grid were used to identify the scale $\mathrm{j}$, where the echoes are separated.
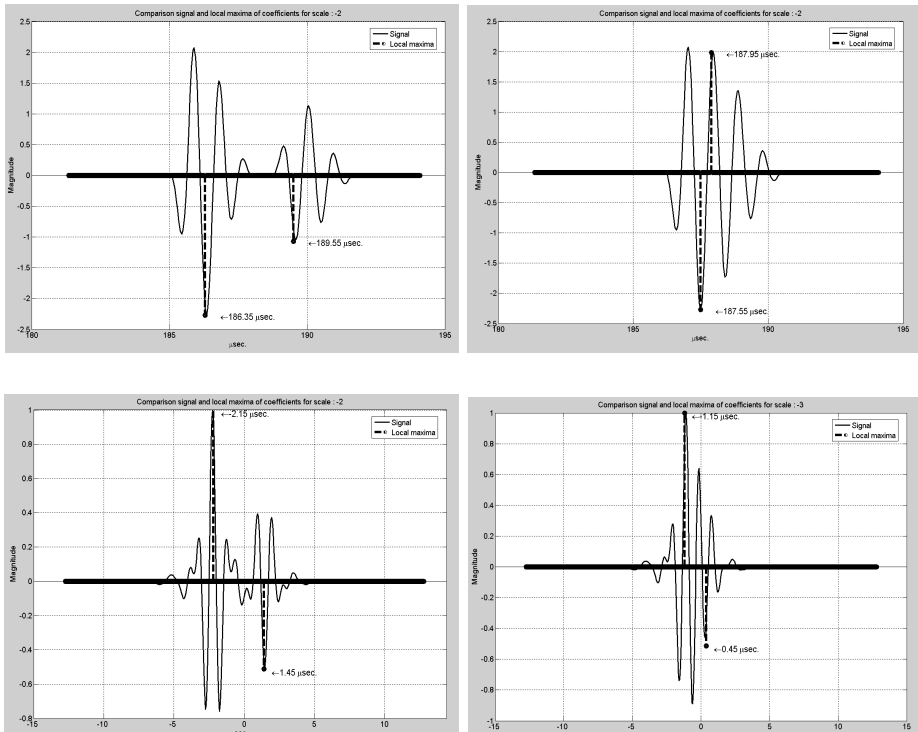

Fig.5. Dyadic grid corresponding to the scale $\mathbf{J}$ of non-zero coefficients (height) pulse echo (bottom), wavelet $\mathrm{J}=-4$ (left) $5 \mathrm{~mm}$, (right) $2 \mathrm{~mm}, 512$ samples, frequency rate $20 \mathrm{MHz}$, velocity of compression waves $=2700 \mathrm{~m} / \mathrm{s}$

Based on the time at which the maximum coefficient occurred in the 1 st group and the time at which the 
maximum coefficient was reached in the 2 nd group, we obtained the following thickness values (Table 1).

Table 1

\begin{tabular}{|c|c|c|}
\hline Real thickness (mm) & \multicolumn{2}{|c|}{ Calculated thickness (mm) (error \%) } \\
Pulse echo & Wavelet \\
\hline 5 & $5.4(8 \%)$ & $4.995(0.099 \%)$ \\
\hline 2 & $3.24(62 \%)$ & $1,89(5.5 \%)$ \\
\hline
\end{tabular}

When the echoes are fairly well separated, it is also possible to discriminate between the - input and output interfaces, and the error was about $8 \%$. With the waveletbased deconvolution method, the error was reduced to $0.099 \%$. At a thickness of $2 \mathrm{~mm}$, the discrimination based on the pulse echo was more difficult (the error was about 62\%), whereas using the wavelet-based deconvolution method, the error at the interface was only about $5.5 \%$.

It is not easy to test this method experimentally

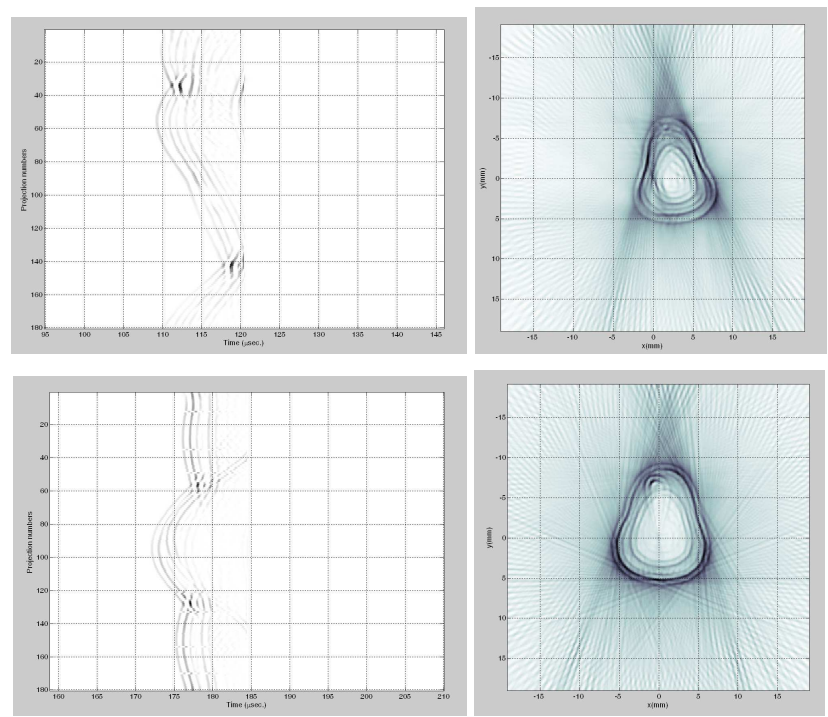

Fig.6. Ultrasonic computed tomography of a non-circular elastic tube. (height) pulse echo (bottom), wavelet $\mathrm{J}=-4$ (left) sinograms, RF-signals, 180 projections through $360^{\circ}$, 1024 samples; (right) tomogram with 300 x 300 pixels.

The surrounding fluid-like medium (and that present in the hollow area) was water at a temperature of $18^{\circ}\left(\rho_{0}\right.$ $\left.=1000 \mathrm{~kg} / \mathrm{m}^{3}, \mathrm{c}_{\mathrm{o}}=1480 \mathrm{~m} / \mathrm{s}\right)$. The transmitter and the receiver were placed $17.5 \mathrm{~cm}$ to the right of the center of the bench. The sinogram drawn up contained 180 projections distributed over $360^{\circ}$ with an angular increment of $2^{\circ}$. The signals contained 1024 distributed samples occurring every $50 \mathrm{~ns}$ (frequency rate of 20 $\mathrm{MHz}$ ). The nominal frequency of the transducer was 1 $\mathrm{MHz}$.

After performing wavelet-based decomposition on the all cross-sectional signals received, the definition of the outer and inner boundaries of the cylinder was obtained with a much better resolution.

\section{E. Aknowledgements}

The author is grateful for the technical assistance and manpower provided by Louise Matet and Laurent Marsac at the Ecole Supérieure des Ingénieurs de Luminy (ESIL) because approaching the numerical transmitted signal $x(t)$ in the form of wavelets makes it necessary to process an initial function, which has to be filtered by the transducer to obtain a specific wavelet. Several approaches were previously tested with various levels of success, such as those based on direct deconvolution and simulated annealing, and especially manual determination, which consists in iteratively producing the signal by correcting the quadratic error at every iteration.

The experimental sample used here was a noncircular homogeneous isotropic tube made of artificial resin (NEUKADUR ProtoCast $113^{\mathrm{TM}}$ ). Its maximum internal and external diameters, which were measured with a caliper, were 6 and $12 \mathrm{~mm}$, respectively. The density of the resin was $\rho_{1} \approx 1150 \mathrm{~kg} / \mathrm{m}^{3}$, and the mean velocity of the compressional wave was $c_{1} \approx 2400 \pm 50$ $\mathrm{m} / \mathrm{s}$. in Marseille and by Karine Degrendele at the Ecole Centrale de Marseille.

\section{F. Literature}

[1] P. Lasaygues, J.-P. Lefebvre and S. Mensah, "High Resolution Low Frequency Ultrasonic Tomography," Ultrasonic Imaging, vol. 19, pp. 278-293, 1997

[2] P. Lasaygues et J.-P. Lefebvre, "Déconvolution en tomographie ultrasonore basse fréquence," Acustica Acta acustica, vol. 86, pp. 506-514 (in French), 2000

[3] P. Lasaygues, Ph-D thesis, University of Aix-Marseille II (in french), 1992

[4] P. Lasaygues et J.-P. Lefebvre, "Application de l'analyse en ondelettes en tomographie ultrasonore," Traitement du Signal, vol. $12 \mathrm{n}^{\circ} 4$, pp. 373-388 (in french), 1995

[5] P. Lasaygues, E. Ouedraogo, J.-P. Lefebvre., M. Gindre, M. Talmant and P. Laugier, "Progress toward in vitro quantitative imaging of human femur using Compound Quantitative Ultrasonic Tomography," Phys. Med. Biol., vol. 50 (11), pp. 2633-2649, 2005 